\title{
Éducation thérapeutique du patient, la révolution est en marche !
}

\author{
La médecine de l'obésité ne veut pas être oubliée !
}

\section{O. Ziegler}

(C) Springer-Verlag 2009

Le concept d'éducation thérapeutique du patient (ETP), auquel est consacré le dossier thématique de ce numéro, n'est pas vraiment nouveau. L'OMS l'a développé, il y a plus de dix ans, et les diabétologues en font depuis plus de 20 ans, sans toujours le savoir. Mais depuis deux ou trois ans, tout va très vite : publications, rapports, recommandations, cahiers des charges, projets de lois ou de décrets s'accumulent.

J.-F. Collin présente une intéressante synthèse sur l'évolution des politiques publiques dans le domaine qui va vous permettre de mieux comprendre les enjeux. Citons, par exemple, le plan 2007-2011 pour «l'amélioration de la qualité de vie des personnes atteintes de maladies chroniques » qui accorde une large place à l'ETP. La HAS a publié, en 2007, des recommandations qui sont remarquables de clarté. Vous trouverez la première dans ce dossier: «Définition, finalités et organisation ». L'inscription de l'ETP dans la loi portant réforme de l'Hôpital, relative aux patients, à la santé et aux territoires (HPST) est un symbole fort, dont on mesure encore mal les conséquences pratiques.

L'obésité, maladie chronique par excellence, est-elle concernée ? La plupart des cliniciens répondraient probablement oui ! Alors, pourquoi est-elle ignorée dans les rapports ? Certains pensent que l'obésité n'est pas une maladie mais simplement un facteur de risque, d'autres que les traitements ne sont pas efficaces ou encore que le corps médical n'est pas prêt à appliquer les règles de bonne pratique...

Peut-être sommes-nous victimes de nos habitudes ou de notre réputation: la seule mise au régime d'un patient obèse, ce n'est pas vraiment de l'ETP !

J.-P. Houppe évoque avec talent le poids des mots et celui de leur histoire pour nous faire partager sa perception de la relation soignant-soigné. Notre vocabulaire a un sens ! Certains mots sont symboliques d'un état d'esprit. Quelle signification donnons-nous à « régime » et à «hygiénodiététique »? Nous savons bien que les régimes sévères, source de restriction cognitive et de troubles du comportement alimentaire, sont peu efficaces à long terme! Certains prônent par conséquent le

O. Ziegler $(\bowtie)$

e-mail : o.ziegler@chu-nancy.fr no diet ! Pourtant, pour perdre du poids ou le stabiliser, il faut bien adapter son alimentation et son activité physique pour que le bilan énergétique soit négatif dans le premier cas ou nul dans le second. Mais ce n'est pas de l'hygiène, que de lutter contre la sédentarité qu'impose notre mode de vie ou notre environnement «toxique ». Les Anglo-Saxons parlent d'ailleurs de « modifications thérapeutiques du mode de vie » (MTMV). Ils n'ont pas tort ! Le poids des mots...

Que peuvent apporter en matière d'ETP les médecins qui soignent les personnes obèses à leurs confrères diabétologues, cardiologues, néphrologues... La médecine de l'obésité se doit d'être une médecine lente, une médecine centrée sur la personne, qui aide le patient à vivre avec une maladie invalidante et difficile à soigner.

Comme le définit A. Grimaldi, «l'ETP constitue, d'une part, un apprentissage pratique et spécialisé pour l'acquisition de compétences thérapeutiques et, d'autre part, une aide au changement de comportement de soin pour atteindre des objectifs personnalisés. Ce n'est pas l'un sans l'autre ». En d'autres termes, on distingue les compétences techniques des compétences psychosociales ou d'adaptation. Or, dans le domaine technique, la personne obèse n'a pas grandchose à apprendre si ce n'est quelques éléments sur le bilan énergétique et la diététique.

L'enjeu véritable est ailleurs. Il s'agit d'apprendre à vivre avec un handicap qui se voit et qui est souvent désespérément rebelle à toute sollicitation thérapeutique. Un handicap somatique qui peut entraver la vie sociale, qui retentit sur le bien être mental et donc immanquablement sur la qualité de vie. L'objet de l'ETP est d'amener le patient à s'engager dans une « démarche de résolution de problème ». Acquérir des compétences psychosociales, c'est avoir la capacité de faire face aux difficultés de sa vie quotidienne (stress, conflits, incidents ou accidents, etc.). C'est pouvoir trouver de nouveaux moyens pour maîtriser son angoisse...

On sort de la technique (autosurveillance glycémique, injections d'insuline, mesure de la tension artérielle) pour entrer dans l'humain.

Le soignant devient un partenaire et un miroir, les spécialistes de l'entretien motivationnel parlent d' "écoute réflective ». L'évaluation de la motivation ou plutôt de 
l'aptitude au changement est essentielle. Les objectifs sont négociés avec le patient, au fur et à mesure de la prise en charge. L'ETP est une sorte de contrat qui conduit le soigné à chercher par lui-même les solutions pour changer sa façon de vivre, c'est-à-dire pour agir sur les différents éléments qui conditionnent son comportement, en s'appuyant sur l'empathie et le savoir-faire du soignant.

Il reste encore beaucoup d'interrogations : comment adapter les concepts de l'ETP à la médecine de l'obésité ?
Faut-il développer des outils spécifiques ? Durrer et Schutz nous font part de leur expérience. Comment l'ETP s'inscritelle dans le parcours de soin du patient ? Comment faire du médecin traitant le copilote de cette démarche, dans le cadre d'une approche multidisciplinaire ? Comment seront financées les actions des uns et des autres ?

Le journal ouvre ses colonnes... Votre point de vue, vos expériences, vos espoirs ou vos craintes dans ce domaine nous intéressent! 